\title{
Memory for detail in item versus associative recognition
}

\author{
ANNE M. CLEARY, TIM CURRAN, and ROBERT L. GREENE \\ Case Western Reserve University, Cleveland, Ohio
}

\begin{abstract}
Some studies have shown that, although repetition increases the familiarity of a stimulus, it does not improve memory for its details. Because memory for associativeinformation is thought to require memory for the details of study presentation, the effects of repetition on associative recognition were examined in the present study. The pattern of results was similar to that found for the recognition of item details: Repetition increased the familiarity of the individual items within each pair to a greater extent than it improved memory for their specific pairings.
\end{abstract}

Repeated exposure to an item or event ordinarily makes it easier to remember later. However, an exception to this general principle occurs when memory for an item's details is considered. Repeated exposure to an item does not greatly improve later discrimination between it and a highly similar item (Hintzman \& Curran, 1995; Hintzman, Curran, \& Oppy, 1992). This finding has been attributed to a tendency for people to base many recognition judgments on overall familiarity and to ignore small details in repetitions (DiGirolamo \& Hintzman, 1997). The experiments reported here demonstrate a similar pattern in associative recognition. The results suggest that, even after pairs of items have been repeated throughout a list, participants often base their associative recognition decisions on the familiarity of the individual items. Furthermore, the familiarity of individual items appears to increase more dramatically with repetition than memory for specific pairings.

Hintzman et al. (1992) gave participants a list containing repeated presentations of singular or plural forms of nouns (e.g., lake, cows, desks, lake). They then tested them for recognition of the same (e.g., lake) or different form (e.g., lakes) with a frequency judgment test. Recognition that a word had changed its singular or plural form from study to test would be shown by a frequency estimate of zero given to each of the different forms. Hintzman et al. (1992) showed that participants' judgments of frequency increased markedly as a function of presentation frequency; this was found both for items that stayed the same and for those that changed plurality. Discrimination between the same- and different-plurality forms improved only slightly over repetitions. Analogous findings were obtained using pictures that either stayed the same

This research was conducted while A.M.C. was supported by the National Institute of Child Health and Human Development Mental Retardation Research Training Grant 5-T32-HDO7176-16. Correspondence should be addressed to A. M. Cleary, Department of Psychology, Case Western Reserve University, 10900 Euclid Ave., Cleveland, OH 44106-7123 (e-mail: amc16@po.cwru.edu). from study to test or changed orientation. Hintzman et al. (1992) labeled this phenomenon registration without learning, because participants were clearly registering the occurrences of the items yet did not appear to be learning more about their details with repeated presentations.

The registration without learning phenomenon suggests that, although repeating an item increases its overall familiarity, its details (e.g., plurality) tend to get ignored in repetitions. DiGirolamo and Hintzman (1997) found evidence supporting this notion. Using pictures that could change in their left or right orientations as stimuli, these researchers found that, when a picture had been repeated in the opposite orientation as that of the first presentation, participants often claimed to have seen all repetitions of the picture in the orientation that was presented first.

Why is it that judgments of frequency to items that change in detail from study to test increase as a function of the frequency of the studied version? Hintzman et al. (1992) argued that above-zero frequency estimates given to changed items are based on their familiarity. Supporting evidence was found by Hintzman and Curran (1994), who showed that participants can make fast recognition judgments if told to ignore the plurality of test items. However, discrimination between items changed and unchanged in plurality was possible only if more time was allowed for the decision; this extra time was presumably used to recollect the details of the original learning episodes.

Like the plurality and picture orientation tasks used by Hintzman et al. (1992) and DiGirolamo and Hintzman (1997), associative recognition (the recognition that a pair of items occurred together in a list) is thought to require memory for detail. In associative recognition, participants typically study a list of word pairs (e.g., candypen, dog-shirt, ball-hat) and then must discriminate between intact (e.g., candy-pen) and rearranged pairs (e.g., dog-hat). Many studies have suggested that, in order to do this accurately, participants must recollect the specific pairings of the words, rather than responding on the basis of familiarity (Clark, 1992; Clark, Hori, \& Callan, 1993; Clark \& Shiffrin, 1992; Hockley \& Consoli, 1999; Yone- 
linas, 1997). For example, it takes participants longer to retrieve associative information than to utilize familiarity in item recognition (Gronlund \& Ratcliff, 1989). Also, associative recognition is more accurate for high-frequency words than for low-frequency words (Clark, 1992; Clark \& Shiffrin, 1992); in this, it resembles free recall more than it does item recognition, which typically shows a low-frequency advantage. When recollection fails, participants may base associative recognition decisions, in part, on the familiarity of individualitems (Kelley \& Wixted, 2001), but this generally would not lead to increased accuracy.

In this series of experiments, we applied the item detail recognition paradigm of Hintzman et al. (1992) to associative recognition. For two reasons in particular, we wanted to determine whether a pattern similar to the registration without learning phenomenon would be shown. First, although previous studies have suggested that recollection plays a more prominent role in associative recognition than does familiarity (e.g., Clark et al., 1993; Yonelinas, 1997), little is known about the effects of repetition on this role. It is not clear whether repetition increases the likelihood that a particular pair will be recollected or whether it increases only the familiarity of the individual items within the pair. The registration without learning phenomenon in item detail recognition (Hintzman et al., 1992) suggests that repetition increases the familiarity of items to a greater degree than it increases the likelihood that their details will be remembered. This is, in part, because frequency estimates are believed to be sensitive to familiarity (e.g., Hintzman, 1988; Hintzman \& Hartry, 1990), and, although both recollection and familiarity may play a role in frequency estimates to unchanged items, estimates that are greater than zero for changed items can be seen as reflecting familiarity (e.g., Hintzman \& Curran, 1994).

Our second reason for applying Hintzman et al.'s (1992) paradigm to associative recognition was to make a comparison between the two tasks. Although both item detail and associative recognition tasks have been used to gather evidence for a recollection process in recognition memory (e.g., Clark et al., 1993; Hintzman \& Curran, 1994; Hockley \& Consoli, 1999; Yonelinas, 1997), little is known about whether the processes involved in these two tasks behave similarly. One way to demonstrate such a similarity would be to take a phenomenon considered critical in one task and show that it can be found in the other. Therefore, we attempted to find the registration without learning phenomenon in associative recognition.

Searching for similarities between these tasks is important, because, although many tasks may be used to demonstrate the existence of recollection in recognition, results obtained for the recollection of one type of information may not generalize to the recollection of other types. If a similar pattern is found in associative recognition, it might suggest that item detail and associative recognition tasks tap into the same type of recollection process and that broad conclusions about the nature of familiarity and recollection can be drawn using either of these tasks. If a different pattern is found, it would suggest that recollection operates differently depending on the type of information being recollected and that caution should be exercised when making generalizations based on results obtained using only one type of task.

There are differences between item detail and associative recognition tasks that might lead one to expect a pattern in associative recognition different from that found by Hintzman et al. (1992). One is that, with Hintzman et al.'s (1992) item detail recognition tasks, the discrimination made at test is binary in nature (i.e., singular/plural or left/right orientation); most associative tasks are not binary in nature because many different repairings among words are possible. Another reason one might expect a different pattern to occur with associative recognition is that the degree to which participants are willing to rely on familiarity may differ. In Hintzman et al.'s (1992) study, participants may have developed a strategy whereby if they did not recollect a particular item, they would respond on the basis of the test item's familiarity. Participants may be less inclined to base associative recognition decisions on the familiarity of individual items than to base item detail recognition decisions on familiarity. This is plausible given that past research has shown that familiarity is more useful for item recognition than for associative recognition (e.g., Yonelinas, 1997) and that participants are at least somewhat aware of this (Hockley \& Consoli, 1999).

In the experiments reported here, two approaches were taken to applying the paradigm of Hintzman et al. (1992) to associative recognition. In the first approach, an associative analog to the plurality and picture orientation tasks was designed. In the second approach, a tactic of converging operations was employed in which the plurality task used by Hintzman et al. (1992) was incorporated in an associative recognition task.

\section{EXPERIMENT 1}

An associative analog to the plurality task used by Hintzman et al. (1992) was used here. To make an associative task comparable to the binary nature of the plurality and picture orientation tasks, rather than pairing each word in the study list with another word, each was paired with one of two letters (e.g., A-lake, or B-candle). The participants were tested with pairs that remained the same from study to test (e.g., A-lake) and with pairs that changed so that the opposite letter was used with the word at test (e.g., A-candle). It should be noted that, although Hintzman and Curran (1995) used a similar paradigm in one experiment, they did not examine judgments of frequency. Therefore, the paradigm was not used to elicit the registration without learning phenomenon as in the present study.

Deciding with which of two letters a word was paired is similar to deciding in which of two lists a word had 
occurred (e.g., List A vs. List B). Like the associative recognition task, list discrimination tasks are thought to require recollection of study episodes (Hintzman, Caulton, \& Levitin, 1998; Jacoby, 1991). It is possible that presenting letter-word pairs together as members of a list (e.g., all of the $A$ pairs together) may make it easier to discriminate between pairs that are the same and those that changed from study to test. Therefore, two methods of study presentation were compared here: a mixed format (e.g., $A$ and $B$ pairs randomly intermixed) and a blocked format (e.g., $A$ pairs presented first, then $B$ pairs).

\section{Method}

Participants. The participants were 114 undergraduates who participated in groups of 1-9 to fulfill a course requirement.

Design and Materials. One hundred twenty words, each from three to seven letters in length, were used as stimuli. Sixty words appeared in the blocked study presentation, and 60 appeared in the mixed study presentation. Because the participants participated in both types of study presentations, the order in which the two occurred was counterbalanced across participants. In addition, so that each group of participants viewed a unique study list, the words used in each experimental session were randomly assigned to their corresponding letters, to their presentation frequencies, and to their presentation conditions (blocked vs. mixed).

Within each study list, words were presented with one of two possible letters. For the first study list, half of the words were paired with the letter $A$ and half were paired with $B$. Words in the second study list were paired with $C$ or $D$. Each letter-word pair was presented $1,3,8$, or 15 times. For the blocked study presentation, the first half of the letter-word pairs contained the same letter (e.g., $C$ ), whereas the latter half contained the opposite letter (e.g., $D$ ). For the mixed study presentation, the letter-word pairs were randomly dispersed throughout the list. Both study lists were separated into two halves by a 1-min break, during which the participants were told to rest before beginning the next segment of the study list. To absorb primacy and recency effects, three buffer pairs were presented at the beginning and end of each study list, as well as before and after each study break.

Each study list was immediately followed by a frequency judgment test. In each of the two test lists, 60 letter-word pairs were presented, 12 of which included words that were not at all studied. Half of the 12 words in each of the four presentation frequency conditions $(1,3,8$, and 15$)$ were paired with the same letter as that with which they were studied, and half were paired with the opposite letter.

Procedure. The participants were seated in chairs surrounding a 21-in. computer monitor. They were told that they would be viewing lists of letter-word pairs and that some pairs would be repeated throughout the study list. They were instructed to pay particular attention to the pairings of the letters and words and to keep track of how many times each specific pair appeared, because they would need to estimate this during testing. The participants were informed beforehand of the type of list they would be viewing (either blocked or mixed format).

All stimuli were presented one pair at a time, in the center of the computer monitor. Each word was presented using lowercase letters, with its corresponding letter centered above it in uppercase form. During study, letter-word pairs were each presented for a duration of $2.5 \mathrm{sec}$ with a 0.5 -sec interstimulus interval (ISI).

During testing, letter-word pairs were each presented for $4 \mathrm{sec}$, with a 1.5 -sec ISI. For each pair presented at test, a number that corresponded to a numbered space on the test sheet was shown in the bottom left corner of the monitor. As each test pair was presented, the participants indicated how many times they thought it appeared in the study list by writing an estimate in the appropriate space. They were instructed to give an estimate of zero to any words paired with a letter different from that at study, as well as to words that did not appear in the study list at all.

\section{Results and Discussion}

In all results reported here, a criterion level of .05 was used to determine significance. More than one dependent measure was derived from the data to determine the effects of presentation frequency on memory. First, judgments of frequency were examined to determine whether presentation frequency had an overall effect on the participants' frequency judgments for pairs that changed ("changed" pairs) and for those that remained the same from study to test ("intact" pairs). The effects of presentation frequency on participants' frequency estimates can be seen in Figure 1. The mean frequency estimates given to the test stimuli that were presented zero times in the study list were .78 and .74 for the mixed and blocked conditions, respectively.

A $2 \times 2 \times 4$ task (blocked vs. mixed) $\times$ test pair (same vs. changed) $\times$ frequency $(1,3,8$, and 15) repeated measures analysis of variance (ANOVA) revealed no three-way interaction $\left[F(3,339)=1.28, M S_{\mathrm{e}}=2.07\right]$. There was no two-way interaction between task and frequency $\left[F(3,339)=0.42, M S_{\mathrm{e}}=2.37\right]$ or between task and test pair $\left[F(3,339)=0.00, M S_{\mathrm{e}}=3.41\right]$. A marginally significant main effect for task on frequency judgments $\left[F(3,113)=3.68, M S_{\mathrm{e}}=5.08, p<.06\right]$ was found. As is apparent in Figure 1, this effect reflects a tendency for the participants to give higher frequency judgments to pairs from the mixed condition than to those from the blocked condition. The interaction between test pair and frequency was significant $\left[F(3,339)=226.87, M S_{\mathrm{e}}=\right.$ 4.17], such that the effect of presentation frequency was larger for judgments given to intact pairs than to changed pairs (see Figure 1).

Although presentation frequency had a greater effect on judgments given to intact pairs than to changed pairs, it had a significant effect on judgments given to both types of test pairs. A one-way repeated measures ANOVA for frequency $(1,3,8$, and 15) on judgments given to intact pairs showed that presentation frequency had a significant effect $\left[F(3,339)=419.38, M S_{\mathrm{e}}=3.29\right]$. Another oneway repeated measures ANOVA on judgments to changed pairs also showed a significant effect of presentation frequency $\left[F(3,339)=31.30, M S_{\mathrm{e}}=1.30\right]$.

Familiarity effects are particularly evident when only the judgments of frequency greater than zero are considered (see Figure 2). Basing recognition decisions on recollection of the specific pair would lead the participants to give an estimate of "zero" to changed pairs. When frequency judgments of zero are excluded from the data, as Figure 2 shows, the participants' frequency estimates for changed pairs very closely track those for intact pairs. Therefore, even though the participants were aware here that the focus of the task was on the specific letter-word 


\section{Mean JOFs for Experiment 1}

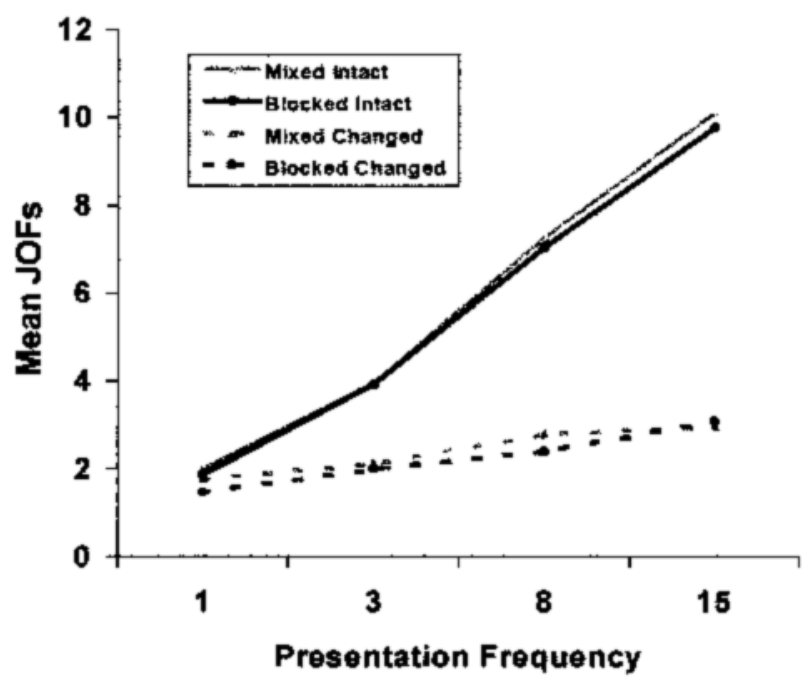

Mean JOFs for Experiment 3

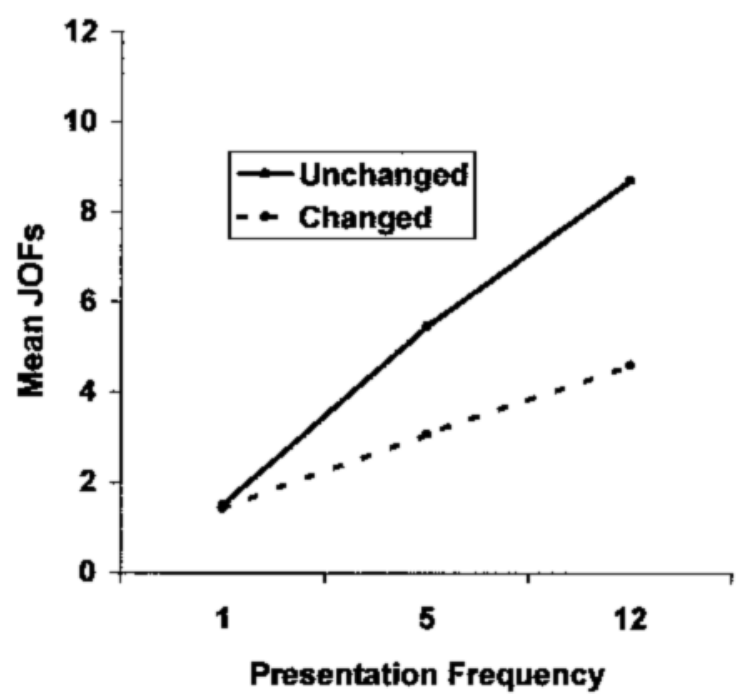

\section{Mean JOFs for Experiment 2}

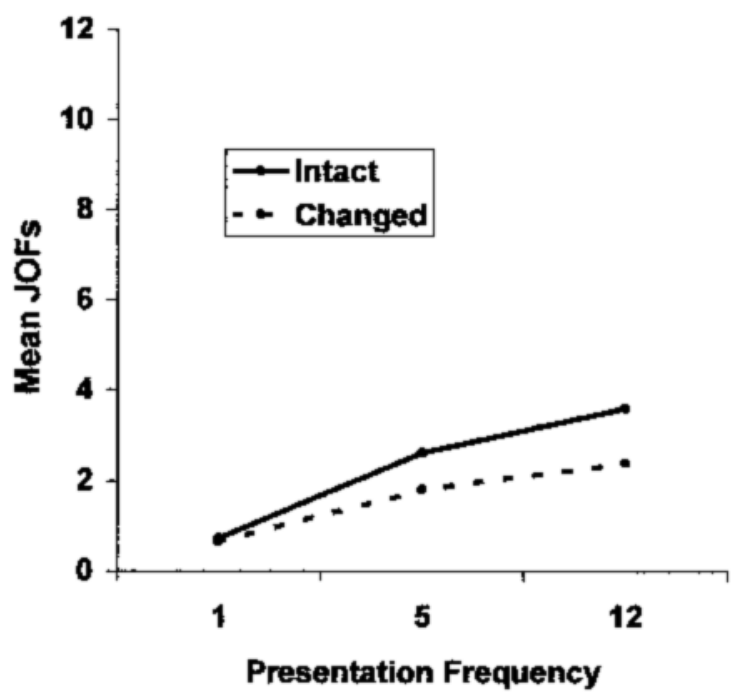

Mean JOFs for Experiment 4

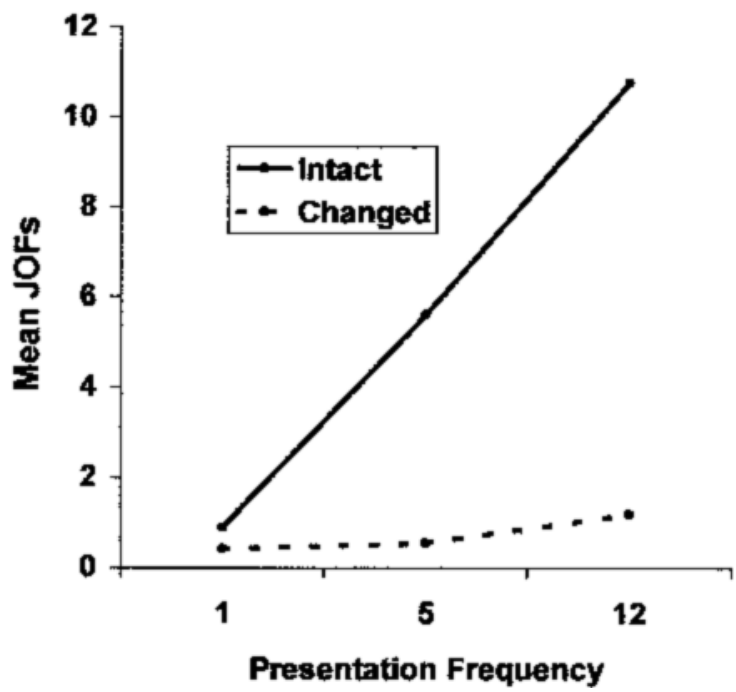

Figure 1. Mean judgments of frequency (JOFs) given during Experiments 1, 2, 3, and 4.

pairings, it appears that they based many of their associative recognition decisions on the familiarity of the individual words.

A second dependent measure derived from the data was the proportion of frequency judgments equal to zero $(\mathrm{JOF}=0)$. This measure served as an indication of whether the participants' ability to discriminate between changed and unchanged pairs improved with repetition. The effects of presentation frequency on the proportion of "zero" judgments that the participants gave are shown in Figure 3. Improved discrimination would be shown if the number of "zero" judgments that the participants gave decreased with presentation frequency for intact pairs and increased for those that changed. Indeed, as can be seen in Figure 3, this appears to be the case.

For changed pairs, a one-way ANOVA for frequency $(1,3,8$, and 15$)$ showed that presentation frequency had a significant effect on the number of "zero" judgments that the participants gave $\left[F(1,339)=16.05, M S_{\mathrm{e}}=0.02\right]$, such that more "zero" judgments were given to changed pairs as presentation frequency increased (see Figure 3). For pairs that remained the same from study to test, a one-way ANOVA again showed that frequency had a significant effect on the number of "zero" judgments that 
Mean JOF $>0$ for Experiment 1

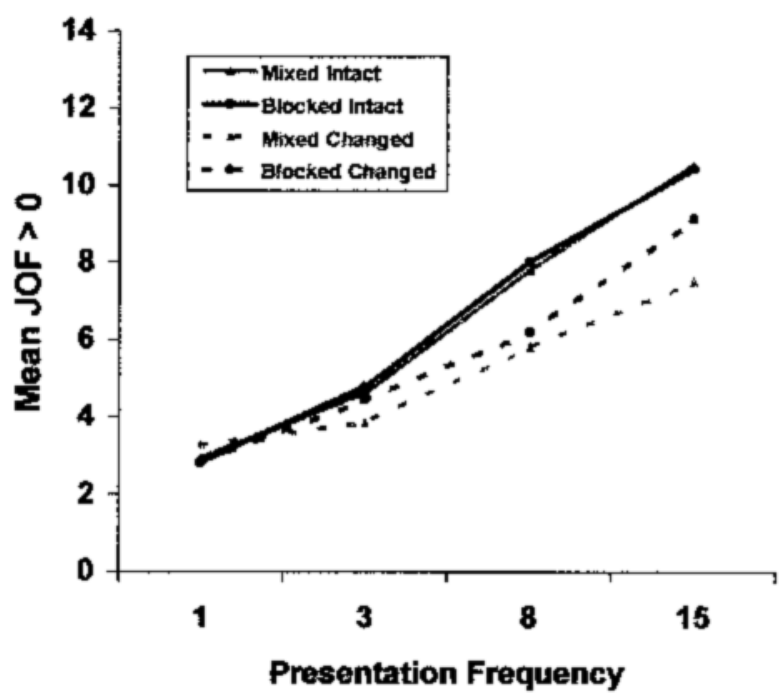

Mean JOF $>0$ for Experiment 3

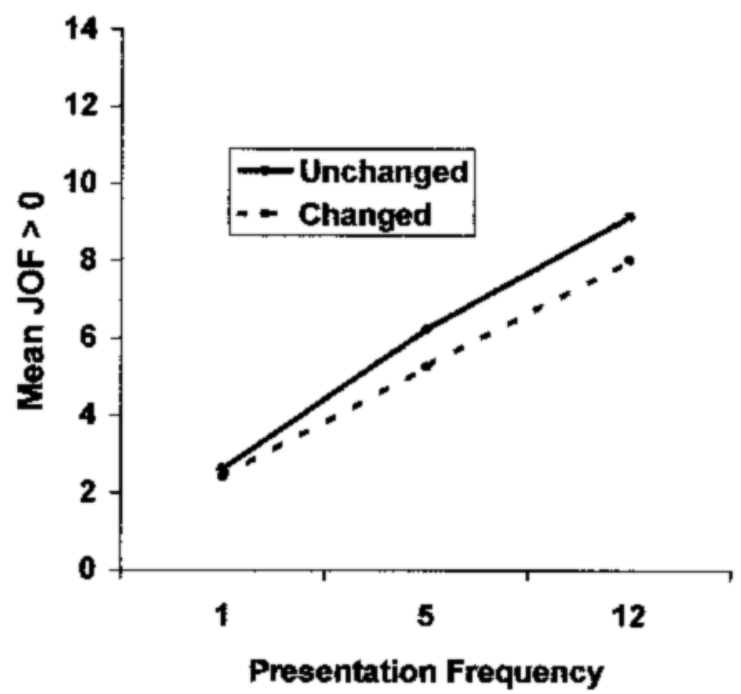

Mean JOF $>0$ for Experiment 2

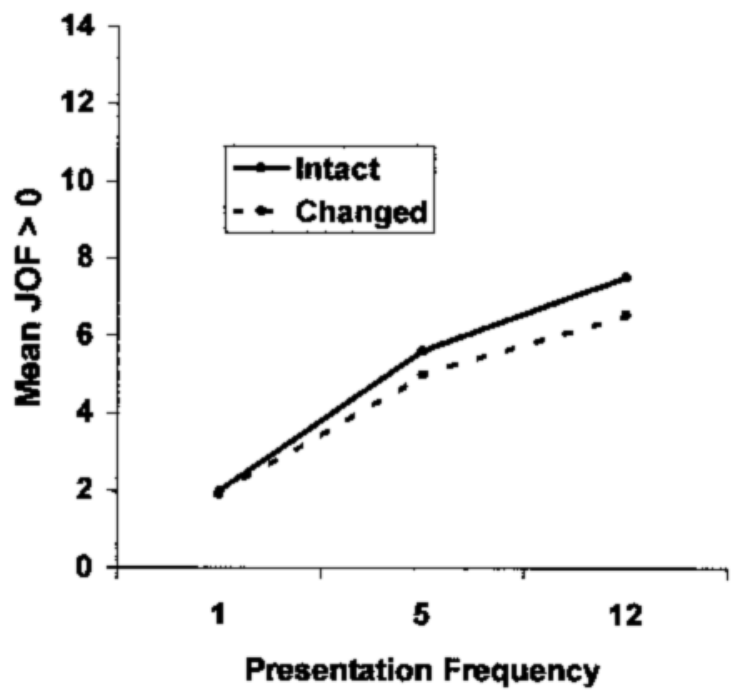

Mean JOF $>0$ for Experiment 4

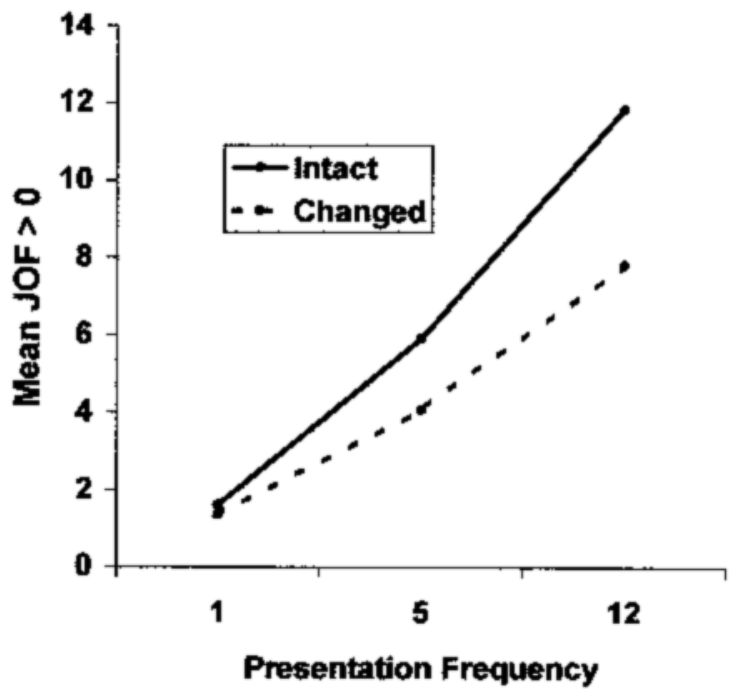

Figure 2. Mean nonzero frequency judgments (JOF $>0$ ) given during Experiments 1, 2, 3, and 4.

the participants gave $\left[F(1,339)=119.08, M S_{\mathrm{e}}=0.01\right]$, only this time such that fewer "zero" judgments were given to intact pairs as frequency increased (see Figure 3). It is worth noting here that the tendency for the participants to accept intact pairs as studied may have been nearing ceiling at the higher presentation frequencies. The proportion of "zero" judgments given to test pairs that were presented zero times in the study list was the same in both conditions (.26 in the mixed condition and .26 in the blocked condition).

The next set of analyses was performed on the proportion of judgments equal to zero to determine whether the type of task (blocked vs. mixed) had any effect on the participants' ability to discriminate between changed and unchanged letter-word pairs. Judgments of frequency for changed and unchanged pairs were analyzed separately. A $2 \times 4$ task (blocked vs. mixed) $\times$ frequency $(1,3,8$, and 15) repeated measures ANOVA for judgments given to changed test pairs showed no significant interaction between task and presentation frequency $[F(3,339)=$ $\left.1.12, M S_{\mathrm{e}}=0.03\right]$. There was a significant main effect $\left[F(1,113)=6.35, M S_{\mathrm{e}}=0.09\right]$, whereby the participants gave more estimates of zero to changed pairs in the blocked condition than in the mixed condition (see Figure 3). For 


\section{$\mathrm{JOF}=0$ for Experiment 1}

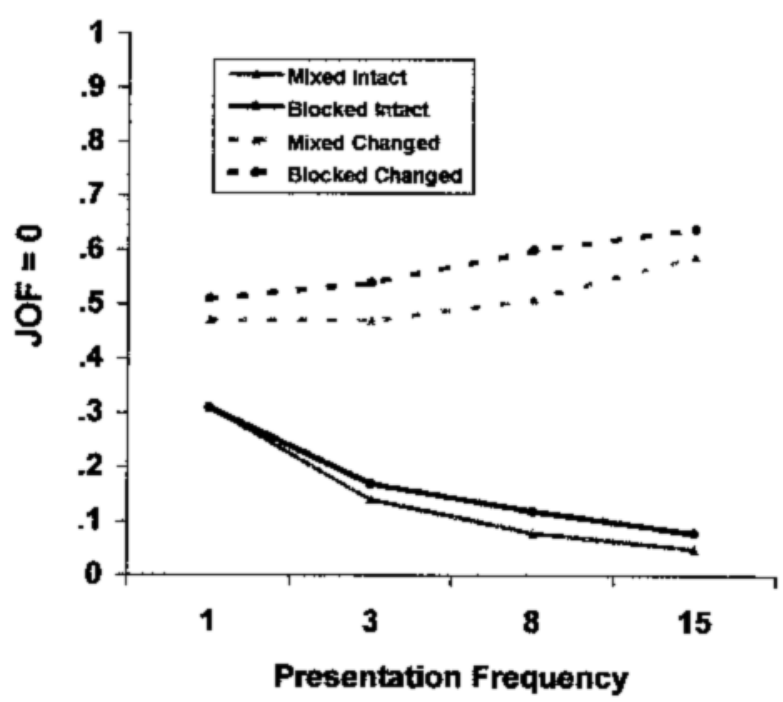

\section{JOF $=0$ for Experiment 3}

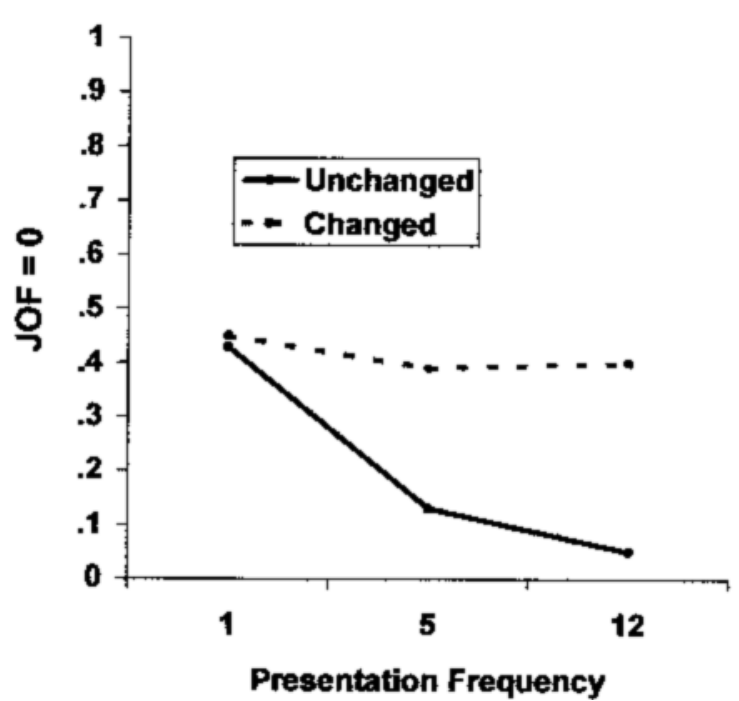

$\mathrm{JOF}=0$ for Experiment 2

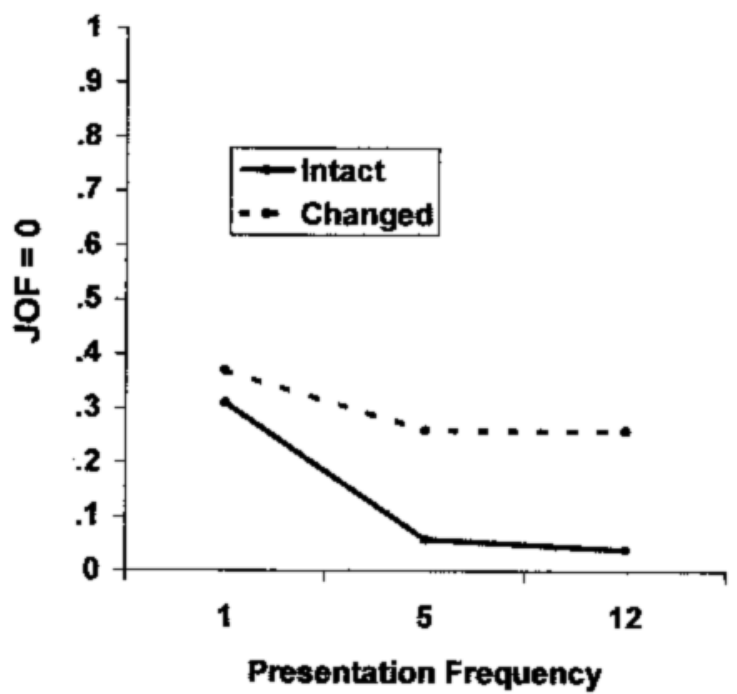

JOF $=0$ for Experiment 4

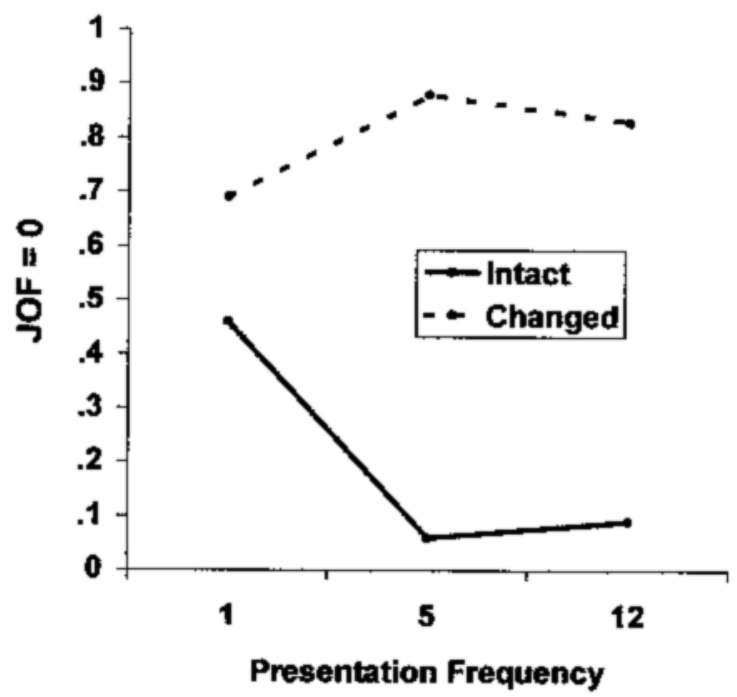

Figure 3. Mean proportions of frequency estimates equal to zero $(\mathrm{JOF}=0)$ that were given during Experiments $1,2,3$, and 4.

intact pairs, there was also no significant interaction revealed by a $2 \times 4$ task $\times$ frequency repeated measures ANOVA on the participants frequency estimates $[F(1,339)$ $\left.=0.20, M S_{\mathrm{e}}=0.02\right]$. However, there was a marginally significant main effect for task $\left[F(1,113)=4.28, M S_{\mathrm{e}}=\right.$ $0.03, p<.06]$, whereby the participants gave more estimates of zero to unchanged pairs in the blocked condition than in the mixed condition. Taken together, the results for the unchanged pairs and the changed pairs reflect an overall bias to give judgments greater than zero more often to pairs in the mixed condition than to those in the blocked condition (see Figure 3).
Overall, the pattern characteristic of registration without learning (Hintzman \& Curran, 1995; Hintzman et al., 1992) was shown in Experiment 1. In particular, abovezero estimates given to changed pairs closely tracked those given to intact pairs, which suggests that when participants do not remember specific pairings of items, they tend to base their associative recognition decisions on the familiarity of the individual items. This is consistent with the findings of Kelley and Wixted (2001), who showed that participants rely on familiarity in associative recognition, even though doing so often hurts their accuracy in discriminating between intact and changed 
pairs. Therefore, one purpose of Experiment 2 was to decrease the likelihood that participants would rely on familiarity to make associative recognition decisions. Another purpose was to explore memory for item detail within an associative recognition task.

\section{EXPERIMENT 2}

Hintzman and Curran (1995) have shown that manipulating the instructions given to participants had little effect on the registration without learning phenomenon in item detail recognition. Therefore, we took a different approach toward reducing the likelihood that the participants would use the familiarity of individual words as a basis for making associative recognition decisions in Experiment 2. A tactic of converging operations was used. For this task, the participants had to pay attention not only to the specific pairings of words but also to the specific singular or plural forms of the words within each pair. For every pair of words presented in the study list (e.g., lake-books, dogs-pencil), a pair containing the same words in their opposite singular or plural forms (e.g., lakes-book, dog-pencils) was presented the same number of times throughout the study list. At test, pairs either were presented in the same manner as at study (e.g., lakebooks, lakes-book) or were re-paired so that one word from each of the opposite pairs were paired together (e.g., dogs-pencils, dog-pencil). Because they would see that both the singular version and the plural version of each word appeared in the study list, the participants were expected to be more aware here that the familiarity of the individual words would not be a useful basis for making associative recognition decisions.

\section{Method}

Participants. The participants were 14 undergraduates who participated individually to fulfill a course requirement.

Design and Materials. Sixty nouns, each pluralizable by adding an $s$, were selected for use in the experiment. Both the singular form and the plural form of each word were used. Through the use of a computer program, 30 word pairs were created by randomly pairing one version of each word with that of another (e.g., cat-bags, pears-nails). The opposite singular or plural versions of the words within each of these pairs were then paired together (e.g., cats-bag, pear-nail), forming 30 more word pairs. Each word pair and its corresponding "opposite" word pair was presented 1 time, 5 times, or 12 times on the study list. The computer program randomly determined both the assignment of pairs to presentation frequencies and the order in which these pairs were presented in a particular study list, so that each participant viewed a unique study list.

The study list was immediately followed by a frequency judgment test. The test list contained 80 word pairs, 20 of which contained words that had not appeared in the study list. Five pairs from each presentation frequency $(1,5$, and 12$)$, along with each of their corresponding opposite word pairs from the study list, were presented in the same manner as at study (e.g., cat-bags and cats-bag). The remaining 10 test pairs from each presentation frequency condition contained re-paired words so that one word from a study pair and one word from its opposite pair were presented together as a pair (e.g., pears-nail, pear-nails). The participants were tested once with a pair containing a specific singular/plural combination of words (e.g., cat-bags) and again with a pair containing the opposite singular/ plural combination (e.g., cats-bag).

Procedure. The participants were told that they would be viewing a list of word pairs and that the words within each pair would vary in terms of whether they would be singular or plural. They were told that they would need to remember specifically which singular or plural versions of words were paired with which versions of other words. They were also told that some of the pairs would be presented more than once and that they would be asked to estimate the number of times they saw each pair in the study list.

During study, each word pair was presented one at a time at the top left corner of the monitor for approximately $2.5 \mathrm{sec}$. The words within each pair were presented side by side. Following presentation of the study list, a self-paced test was given. For each test pair, the participants were instructed to type in the number of times they remembered seeing the pair in the study list. They were told that many test pairs would contain words from the study list that were not studied together as a pair. They were instructed not to estimate the frequencies of the individual words but to estimate the frequencies of the pairs as a unit and to give estimates of zero to any pairs that did not appear together in the study list. In addition, they were asked to pay particular attention to whether each test pair contained the same singular or plural versions of the words that were seen in a pair during the study list and to give an estimate of zero to any that did not. They were also asked not to give any estimates greater than 15 .

\section{Results and Discussion}

As with Experiment 1, more than one dependent measure was derived from Experiment 2. Judgments of frequency were examined first to determine the effects of presentation frequency on participants' frequency estimates (see Figure 1), followed by an analysis of the proportion of "zero" judgments given (see Figure 3). The mean frequency estimate given to test pairs that appeared zero times in the study list was .13. The proportion of zero judgments given to such test pairs was .41.

For every test pair presented, the participants were later given a highly similar test pair (e.g., cat-bags then cats-bag). The data were first analyzed to determine whether this affected frequency judgments. There were no significant main effects of order (first pair vs. second pair) on either dependent measure. One significant interaction was found. A $2 \times 2 \times 3$ order (first pair vs. second pair) $\times$ test pair (same vs. changed) $\times$ frequency $(1$, 5 , and 12) repeated measures ANOVA found a significant two-way interaction between order and frequency on the participants' judgments of frequency $[F(2,26)=$ $\left.3.97, M S_{\mathrm{e}}=11.31\right]$. This effect was such that frequency estimates increased more with repetition for pairs that were tested second than with repetition for those that were tested first.

In terms of how presentation frequency influenced the participants' estimates for intact and changed test pairs, the two-way interaction between test pair (same vs. changed) and frequency $(1,5$, and 12) was significant $[F(2,26)=$ $\left.6.02, M S_{\mathrm{e}}=75.96\right]$. Although the effect of presentation frequency appears to have been larger for judgments given to intact pairs than for judgments given to changed pairs (see Figure 1), presentation frequency had a significant effect on judgments given to both types of test pairs. A one-way repeated measures ANOVA for frequency on 
judgments given to intact pairs showed that presentation frequency had a significanteffect $\left[F(2,26)=68.30, M S_{\mathrm{e}}=\right.$ 174.37], as did another one-way ANOVA on judgments to changed pairs $\left[F(2,26)=23.91, M S_{\mathrm{e}}=184.21\right]$.

Even though the participants should have been aware in this case that nearly all of the words at test would be familiar and, therefore, that the familiarity of individual words would not be useful, they still often used familiarity as a basis for making frequency judgments. The tendency for the participants to base associative recognition decisions on familiarity is even more apparent when only judgments of frequency greater than zero are considered. As can be seen in Figure 2, when judgments of zero are excluded, the participants' frequency estimates for changed pairs very closely track those for unchanged pairs.

The effects of presentation frequency on the proportion of frequency judgments that the participants gave that were equal to zero (JOF $=0$ ) are shown in Figure 3. Again, this measure served as an indication of whether the participants' ability to discriminate between intact and changed pairs improved with repetition. Improved discrimination would be shown if the number of "zero" judgments that the participants gave decreased with presentation frequency for intact pairs and increased for changed pairs.

For changed pairs, a one-way ANOVA for frequency $(1,5$, and 12$)$ showed no significant effect on the number of "zero" judgments that the participants gave $[F(2,26)=$ $\left.1.99, M S_{\mathrm{e}}=3.07\right]$. A one-way ANOVA showed that frequency had a significant effect on the number of "zero" judgments that the participants gave to pairs that were the same from study to test $\left[F(2,26)=21.14, M S_{\mathrm{e}}=\right.$ $0.02]$; again, it appears that the tendency for the participants to accept intact pairs as having been studied may have been nearing ceiling at the presentation frequency of 12 . However, the ability to reject changed pairs was not near ceiling and did not appear to have improved with repetition. As can be seen in Figure 3, repetition did not improve discrimination much beyond the 5 th repetition. This finding is consistent with the finding of Hintzman and Curran (1995) and Hintzman et al. (1992) that repetitions beyond the first few presentations do not generally help discrimination.

Increasing the difficulty of the task in Experiment 2 (by asking the participants to remember which singular or plural words were paired with which other singular or plural words) does not appear to have changed the overall pattern of results from those found in Experiment 1. That is, the participants still based many of their frequency judgments on the familiarity of the individual words within a given pair. In addition, they do not appear to have improved at giving estimates of zero to changed pairs. It could be that the difficult nature of the task does spur participants to attend more to the details of study presentation but that the task is so difficult that participants simply cannot get any better with repetition. Therefore, in Experiment 3, an item version of the task used in Experiment 2 was employed, whereby the study instruc- tions were the same as those used in Experiment 2, but the nature of the recognition test was made easier.

\section{EXPERIMENT 3}

An item, rather than an associative, version of the task used in Experiment 2 was employed in Experiment 3. For this task, the participants viewed a list of word pairs containing nouns in either their singular forms or their plural forms (e.g., cat-lakes, table-flower) and, as in Experiment 2, were instructed to remember specifically which singular or plural words were paired with which other singular or plural words. However, unlike in Experiment 2, only one version of each word was shown at study, and, for each question at test, the participants were only asked to decide whether or not a particular singular or plural word had occurred in the study list. Some of these words remained the same from study to test (e.g., cat), whereas others changed from singular to plural or from plural to singular from study to test (e.g., flowers).

\section{Method}

Participants. The participants were 14 undergraduates who participated individually to fulfill a course requirement.

Design and Materials. The design and materials used in Experiment 3 were the same as those used in Experiment 2, with the exception that, since an item, rather than an associative, recognition test would be used, only one version (singular or plural) of a given word was used during study. Thirty word pairs were created by randomly pairing one version of each word with that of another (e.g., cat-bags, pears-nails). Each word pair was presented 1 time, 5 times, or 12 times throughout the study list. As in Experiment 2, the computer program randomly determined both the assignment of pairs to presentation frequencies and the presentation order of the stimuli, so that each participant viewed a unique study list.

The study list was immediately followed by a frequency judgment test. The test list contained a total of 80 words, each of which was presented one at a time. Ten of the words from each presentation frequency $(1,5$, and 12) were presented in the same manner as at study (e.g., cat, nails). The remaining 10 words from each presentation frequency were changed to the opposite singular or plural versions than that seen at study (e.g., bag, pear). In addition, 20 test words had not appeared in the study list.

Procedure. The procedure was the same as that used for Experiment 2, with the exception that, at test, the participants were instructed to estimate the frequencies of individual words, rather than pairs of words. They were specifically instructed to give estimates of zero to any words that did not appear in exactly the same form as at study.

\section{Results and Discussion}

As with Experiments 1 and 2, judgments of frequency were examined first. The effects of presentation frequency on the participants' estimates are shown in Figure 1 . The mean frequency estimate to test items that appeared zero times in the study list was .55 . A $2 \times 3$ test word (same vs. changed) $\times$ frequency $(1,5$, and 12$)$ repeated measures ANOVA on judgments of frequency revealed a significant two-way interaction $[F(2,26)=20.24$, $\left.M S_{\mathrm{e}}=141.68\right]$. As with the previous experiments, although presentation frequency had a more dramatic effect on judgments given to unchanged words than on 
judgments to changed words (see Figure 1), a significant effect was found for judgments given to both types of test words. A one-way repeated measures ANOVA for frequency $(1,5$, and 12$)$ on judgments given to unchanged words showed that presentation frequency had a significant effect $\left[F(2,26)=107.06, M S_{\mathrm{e}}=1.70\right]$. Another one-way repeated measures ANOVA for frequency $(1,5$, and 12) on judgments given to changed words also showed a significant effect of presentation frequency $[F(2,26)=$ 13.90, $\left.M S_{\mathrm{e}}=2.51\right]$. As with Experiments 1 and 2, when only judgments of frequency greater than zero are considered, frequency estimates for changed words closely track those of unchanged words (see Figure 2).

The proportion of frequency judgments equal to zero $(\mathrm{JOF}=0)$ was used as an indication of whether the participants' ability to discriminate between changed and unchanged words improved with repetition (see Figure 3 ). The proportion of "zero" judgments given to items that appeared in the study list zero times was .78. For words that changed from study to test, a one-way ANOVA showed that presentation frequency did not have a significant effect on the number of "zero" judgments that the participants gave $\left[F(2,26)=0.31, M S_{\mathrm{e}}=0.04\right]$. A one-way ANOVA showed that frequency had a significant effect on the number of "zero" judgments that the participants gave to unchanged words $\left[F(2,26)=52.70, M S_{\mathrm{e}}=\right.$ 0.01 ], although, as in Experiment 2, the tendency to accept unchanged items as having been studied may have been nearing ceiling at the presentation frequency of 12 . From Figure 3, it is apparent that these results are similar to those found in Experiment 2 and to those of Hintzman and Curran (1995) and Hintzman et al. (1992). Taken together, the results of Experiments 2 and 3 extend the findings of Kelley and Wixted (2001) by suggesting that, even when the task at hand explicitly requires memory for the details of study presentation, whether memory for item or associative information is required at test, participants will often rely on familiarity to make recognition decisions.

\section{EXPERIMENT 4}

The results thus far suggest not only that participants base many of their associative recognition decisions on the familiarity of individual items but that the overall registration without learning pattern found by Hintzman et al. (1992) in item detail recognition may extend to associative recognition as well. One question that remains is whether these findings can be extended to the standard associative recognition task. In other words, would the same general pattern be found when participants must remember only the pairings of items, without having to remember the details of the items within a given pair?

This question was explored in Experiment 4. The participants studied a list of pairs containing nouns in either their singular forms or their plural forms (e.g., cat-lakes, table-flower, books-candle) and were instructed to remember specifically which singular or plural words were paired with other singular or plural words. However, for each pair presented at test, the plurality of the words remained unchanged. The participants were only asked to decide whether or not a particular pair of words, irrespective of plurality, had appeared in the study list. Some of the word pairs remained the same from study to test (e.g., cat-lakes), whereas other words were re-paired from study to test (e.g., table-candle).

\section{Method}

Participants. The participants were 16 undergraduates who participated in fulfillment of an introductory psychology course requirement.

Design and Materials. The design and materials used in Experiment 4 were the same as those in Experiment 3, with the exception of the test list. The test list contained 40 word pairs. Five of the pairs from each presentation frequency $(1,5$, and 12) remained unchanged from study to test. The remaining 5 pairs from each presentation frequency condition were re-paired, so that each word from a given frequency condition was paired with a different word from the same frequency condition at test. In addition, 10 test pairs had not appeared in the study list.

Procedure. The procedure was the same as that of Experiment 2, with the exception that, at test, the participants were instructed to ignore plurality when estimating the number of times they remembered seeing each test pair in the study list. They were told to give estimates of zero to re-paired words and to pairs containing new words.

\section{Results and Discussion}

Judgments of frequency were examined first. The effects of presentation frequency on participants' estimates are shown in Figure 1. The mean frequency estimates to test pairs that appeared zero times in the study list was .06. A $2 \times 3$ test pair (same vs. changed) $\times$ frequency $(1,5$, and 12$)$ repeated measures ANOVA on judgments of frequency revealed a significant two-way interaction $\left[F(2,30)=84.24, M S_{\mathrm{e}}=49.02\right]$. As with the previous experiments, although presentation frequency had a more dramatic effect on judgments given to intact word pairs than to changed word pairs (see Figure 1), frequency influenced judgments to both types of test pairs. A one-way repeated measures ANOVA for frequency on judgments given to intact pairs showed that presentation frequency had a significant effect $[F(2,30)=169.94$, $\left.M S_{\mathrm{e}}=2.30\right]$. A one-way repeated measures ANOVA on judgments to changed pairs showed a marginally significant effect of presentation frequency $[F(2,30)=2.60$, $\left.M S_{\mathrm{e}}=1.10, p<.10\right]$. When only judgments of frequency greater than zero are considered, frequency estimates to changed pairs track those to intact pairs (see Figure 2).

Again, the proportion of frequency judgments equal to zero $(\mathrm{JOF}=0)$ was used as an indication of whether the participants' ability to discriminate between intact and changed word pairs improved with repetition. These data are shown in Figure 3. The proportion of "zero" judgments given to the test pairs that occurred zero times in the study list was .94. For changed pairs, a one-way ANOVA showed a significant effect of frequency $(1,5$, and 12$)$ on the number of "zero" judgments that the participants gave $\left[F(2,30)=3.35, M S_{\mathrm{e}}=0.05\right]$. A second one-way ANOVA 
showed that there was a significant effect of frequency $(1,5$, and 12$)$ on the number of "zero" judgments that the participants gave to intact pairs $[F(2,30)=21.14$, $\left.M S_{\mathrm{e}}=0.04\right]$. As is apparent in Figure 3, however, the effect of presentation frequency primarily occurred between the frequencies of 1 and 5, and not between 5 and 12. It is possible that this finding resulted from a ceiling effect, since discrimination between intact and changed pairs was already quite good at the presentation frequency of 5.

The results of Experiment 4 suggest that the general pattern observed in Hintzman et al.'s (1992) study and in Experiments 1, 2, and 3 may extend to the standard associative recognition task. Above-zero frequency estimates for changed pairs closely track those for intact pairs, whereas discrimination does not improve beyond the 5th repetition of a given pair. However, it should be noted that participants appear to be very good at making the discrimination between intact and changed pairs in standard associative recognition. As can be seen when the graph for Experiment 4 is compared with the graphs from Experiments 1, 2, and 3 in Figure 3, participants are much better at making this discrimination in the standard associative recognition task than they are in the other recognition tasks used in the present study; this occurred despite the fact that the study instructions were the same across Experiments 2, 3, and 4. The finding that discrimination does not improve beyond the first few repetitions of a pair in Experiment 4 may in fact be due to ceiling effects in performance.

\section{GENERAL DISCUSSION}

\section{Overview of the Experiments}

Item detail recognition tasks (e.g., plurality) and associative recognition have played an important role in demonstrating that people use the recollection of specific study episodes in recognition. Although both of these tasks have been shown to involve a greater role for recollection than for familiarity (e.g., Clark et al., 1993; Hintzman \& Curran, 1994; Yonelinas, 1997), little research has been devoted to showing any similarity between these two tasks. In addition, although the effects of repetition on recollection have been examined using item detail recognition tasks (Hintzman \& Curran, 1995; Hintzman et al., 1992), it has not yet been made clear what effect repetition has on the recollection of associative information. In the present study, we addressed both of these issues by examining the registration without learning phenomenon, often studied using item detail recognition tasks (Hintzman \& Curran, 1995; Hintzman et al., 1992), and applying the paradigm to associative recognition.

Two different approaches were taken. In Experiment 1, an associative analog to the plurality and picture orientation tasks used by Hintzman et al. (1992) was used to assess the effects of repetition on binary pair recognition (e.g., whether a word was paired with the letter $A$ or the letter $B$ ). The results were similar to those found by Hintz- man et al (1992). The participants' frequency estimates increased with repetition, not only for letter-word pairs that remained the same from study to test but also for words that were paired with a different letter at test.

In the second approach, the plurality task used by Hintzman et al. (1992) and an associative recognition task were incorporated into one task, for which the participants had to remember specifically which singular and plural versions of a word were paired with which singular and plural versions of another (Experiment 2). Although this task was particularly difficult for the participants, they still relied heavily on the use of familiarity, as evidenced by the fact that frequency estimates continued to rise for changed pairs with repetition. This pattern extended to both an item version of the aforementioned associative recognition task (Experiment 3), whereby the participants based many of their recognition decisions on the familiarity of each word (having ignored the plurality of these words in repetitions), and, to some extent, a more typical associative recognition task (Experiment 4). However, the participants were much better at discriminating between intact and changed pairs in the typical associative recognition task than in the other associative tasks used in the present study.

\section{Dissociating Frequency Estimates and Discrimination}

The biggest empirical generalization across experiments is that the participants' ability to discriminate between intact and changed pairs (as shown by the tendency to give estimates of zero to changed as opposed to intact pairs) increased less dramatically with repetition than did their memory for the occurrence of items (as shown by the rise in frequency judgments to both intact and changed pairs). Thus, the pattern characteristic of registration without learning was observed in all four experiments. Several additional analyses were carried out to demonstrate the reliability of this pattern. Insofar as the pattern only deals with repetitions beyond the first few, only the two highest presentation frequencies used in each experiment were included in the analyses.

To see whether presentations were being registered, analyses were done to determine whether frequency estimates given to pairs (both intact and changed) increased from the second-highest frequency to the highest frequency in each experiment. To see whether learning of details was taking place, a discrimination measure was calculated as the difference between the proportion of estimates of zero given to changed pairs and the proportion given to intact pairs; this discrimination measure was then analyzed to determine whether it increased from the second-highest frequency to the highest frequency.

The claim that frequency judgments were rising as a function of frequency more reliably than discrimination was supported in three ways. First, when significance tests were carried out on differences between the secondhighest frequency and the highest frequency, significant effects were found on estimates in all four experiments 
$\left[F(1,113)=210.44, M S_{\mathrm{e}}=16.91\right.$, in Experiment $1 ; F(1,13)$ $=18.10, M S_{\mathrm{e}}=385.44$, in Experiment $2 ; F(1,13)=$ $22.20, M S_{\mathrm{e}}=715.58$, in Experiment $3 ; F(1,15)=96.68$, $M S_{\mathrm{e}}=70.19$, in Experiment 4]. In contrast, on the discrimination measure, a significant increase was found in Experiment 1 but not in the other experiments $[F(1,113)=$ $22.97, M S_{\mathrm{e}}=2.44$, in Experiment $1 ; F(1,13)=0.29$, $M S_{\mathrm{e}}=1.09$, in Experiment $2 ; F(1,13)=1.40, M S_{\mathrm{e}}=$ 3.68 , in Experiment $3 ; F(1,15)=0.68, M S_{\mathrm{e}}=1.66$, in Experiment 4]; indeed, there was a slight, nonsignificant trend for decreased discrimination as a function of repetition in Experiment 4.

Second, when effect sizes were calculated using Cohen's $d$ to compare the difference between the second-highest frequency and the highest frequency, the effect sizes on the estimates were consistently higher on the judgments of frequency (the values for $d$ were 1.36, 1.18, 1.31, and 2.54 in Experiments 1, 2, 3, and 4, respectively) than on the discrimination measure (the values for $d$ were 0.45 , $0.15,0.50$, and -0.21 in Experiments 1, 2, 3, and 4, respectively; the negative sign indicates that discrimination decreased as a function of frequency in Experiment 4).

Third, when one directly compares the effect sizes found in each experiment, the increase from second-highest frequency to highest frequency had a significantly greater effect on the judgments of frequency than on the discrimination measure in all four experiments $(z \mathrm{~s}=6.87$, 3.29, 2.23, and 2.82 in Experiments 1, 2, 3, and 4, respectively; in each case, $p<.05$ ).

In short, it seems clear that, although presentations continued to be registered at the higher frequencies as shown by significant increases in judgments of frequency, there was, at best, a smaller and less consistent increase in discrimination. That is not to say that the participants were not learning about details over the course of each experiment. The fact that, in all four experiments, the analyses of frequency estimates showed an interaction between the test pair variable and presentation frequency (such that estimates to intact pairs increased more dramatically with repetition than did estimates to changed pairs) suggests that people do learn about the details of stimuli as they are repeated. The argument we are making is more limited-namely, that people learn less about the details of a stimulus that has been repeated many times than they learn about its broader characteristics.

The present results are not inconsistent with the claim that there is a stronger recollective component to associative recognition than familiarity (e.g., Clark et al., 1993; Hockley \& Consoli, 1999; Yonelinas, 1997). Our results only suggest that the two components (recollection and familiarity) are influenced differently by repetition and that, when participants fail to recollect a specific pair, they will tend to rely on the familiarity of the individual words as basis for making their decisions.

\section{Conclusions}

One conclusion that can be drawn from the present results is that similar processes are involved in item detail and associative recognition. The recollection of both types of information seems to respond similarly to repetition. Furthermore, in neither case does an increased awareness of the need to rely on recollection lead to an increased tendency to recollect study episodes with repetition. Even when aware of the need to recollect, when they fail to do so, people resort to using familiarity as a basis for making recognition decisions in both item detail and associative recognition tasks.

\section{REFERENCES}

Clark, S. E. (1992). Word frequency effects in associative and item recognition. Memory \& Cognition, 20, 231-243.

Clark, S. E., Hori, A., \& Callan, D. E. (1993). Forced-choice associative recognition: Implications for global-memory models. Journal of Experimental Psychology: Learning, Memory, \& Cognition, 19, 871-881.

Clark, S. E., \& Shiffrin, R. M. (1992). Cuing effects and associative information in recognition memory. Memory \& Cognition, 20, 580-598.

DiGirolamo, G. J., \& Hintzman, D. L. (1997). First impressions are lasting impressions: A primacy effect in memory for repetitions. Psychonomic Bulletin \& Review, 4, 121-124.

Gronlund, S. D., \& Ratcliff, R. (1989). Time course of item and associative information: Implications for global memory models. Journal of Experimental Psychology: Learning, Memory, \& Cognition, 15, 846-858.

HintzMan, D. L. (1988). Judgments of frequency and recognition memory in a multiple-trace memory model. Psychological Review, 95, 528-551.

Hintzman, D. L., Caulton, D. A., \& Levitin, D. J. (1998). Retrieval dynamics in recognition and list discrimination: Further evidence of separate processes of familiarity and recall. Memory \& Cognition, 26, 449-462.

Hintzman, D. L., \& Curran, T. (1994). Retrieval dynamics of recognition and frequency judgments: Evidence for separate processes of familiarity and recall. Journal of Memory \& Language, 33, 1-18.

Hintzman, D. L., \& Curran, T. (1995). When encoding fails: Instructions, feedback, and registration without learning. Memory \& Cognition, 23, 213-226.

Hintzman, D. L., Curran, T., \& Oppy, B. (1992). Effects of similarity and repetition on memory: Registration without learning? Journal of Experimental Psychology: Learning, Memory, \& Cognition, 18, 667680

Hintzman, D. L., \& Hartry, A. L. (1990). Commensurability in memory for frequency. Journal of Memory \& Language, 29, 501-523.

Hockley, W. E., \& Consoli, A. (1999). Familiarity and recollection in item and associative recognition. Memory \& Cognition, 27, 657664

JACOBY, L. L. (1991). A process dissociation framework: Separating automatic from intentional uses of memory. Journal of Memory \& Language, 30, 513-541.

KELLEY.R., \& WIXTED, J. T. (2001). On the nature of associative information in recognition memory. Manuscript submitted for publication.

YonelinAs, A. P. (1997). Recognition memory ROCs for item and associative information: The contribution of recollection and familiarity. Memory \& Cognition, 25, 747-763.

(Manuscript received August 11, 1999; revision accepted for publication September 11, 2000.) 